\title{
Organizadores da cultura: delimitação e formação
}

Antonio Albino Canelas Rubim*

Pós-doutor em Políticas Culturais pela Universidade de Buenos Aires e Universidade San Martin. Doutor em Sociologia pela Universidade de São Paulo. Professor titular da Universidade Federal da Bahia, onde é docente do Programa Multidisciplinar de Pós-Graduação em Cultura e Sociedade e do Programa de Artes Cênicas. Diretor do Instituto de Humanidades, Artes e Ciências da UFBA. Presidente do Conselho Estadual de Cultura da Bahia e ex-coordenador do Centro de Estudos Multidisciplinares em Cultura. Pesquisador I-A do CNPq.

E-mail: rubim@ufba.br

Lindinalva Rubim**

Pós-doutora pela Universidade de Buenos Aires. Doutora em Comunicaşão pela Universidade Federal do Rio de Janeiro. Graduada em Jornalismo pela Universidade Federal da Bahia. Professora Adjunta IV da Universidade Federal da Bahia. Coordenadora do Centro de Estudos Multidisciplinares em Cultura. Tem experiência na área de Comunicação, com ênfase em Cinema e Televisão, atuando principalmente nos seguintes temas: cultura, comunicação, gênero, cinema, televisão e representações.

E-mail:lrubim@ufba.br

Resumo: O texto discute a relação entre sistema cultural e demanda de novos profissionais na área da cultura, e analisa a configuração de uma área de atuação profissional, a organização da cultura, apontando seus momentos constitutivos: a formulação de políticas, a gestão e a produção cultural. Após refletir sobre as peculiaridades da constituição do campo no País, o texto propõe medidas visando a um desenvolvimento desejável para a formação de organizadores da cultura.

Palavras-chave: organização da cultura, formação em cultura, políticas culturais, gestão cultural, produção cultural, cultura.
Abstract: The subject of education of cultural managers or, more broadly, professionals dedicated to the organization of culture, as will be further examined, today is fundamentally important not only to the development of culture in Brazil and in the world but also to the creation of cultural policies effectively imaginative and contemporary. A deeper analysis of the theme requires some reflection, although a bit panoramic, about culture present configuration; about the organization of a cultural system; and about the singularities of the Brazilian case in this field.

Keywords: culture organization, culture formation, cultural system, cultural production, culture.

Uma reflexão sobre a organização da cultura no mundo e no Brasil contemporâneos pode tomar como ponto de partida uma apropriação livre das formulações de Antonio Gramsci¹, em seu estudo sobre o tema dos intelectuais. Ele, além da sempre lembrada distinção entre intelectuais tradicionais e

1. GRAMSCl, Antonio. A formação dos intelectuais. Venda Nova: M. Rodrigues Xavier, 1972; Os intelectuais e a organização da cultura. Rio de Janeiro: Civilização Brasileira, 1978; Literatura e vida nacional. Rio de Janeiro: Civilização Brasileira, 1978. 
comunicação \& educação • Ano XIV • Número 2 • maio/ago 2009

orgânicos, elabora outros três tipos: aqueles que criam, os que transmitem e difundem a cultura e, por fim, aqueles que a organizam.

Os três tipos de intelectuais podem ser considerados imprescindíveis para a existência de um sistema cultural, ainda que, muitas vezes, em um conhecimento impregnado pelo senso comum, apenas o primeiro tipo - o criador - seja nomeado com o termo intelectual. Cabe lembrar que, para Antonio Gramsci, todo ser humano é potencialmente intelectual, mas apenas alguns desempenham socialmente tal atividade. Por conseguinte, um sistema cultural necessariamente comporta, pelo menos, três momentos e movimentos imanentes: a criação, a divulgação ou transmissão e a organização da cultura.

Um sistema cultural em tempos remotos não demandava a diferenciação destas instâncias. Muito provavelmente, estavam imersas no bojo de um conjunto indiferenciado, que abarcava simultaneamente diversas dessas dimensões. A distinção das atividades faz parte, portanto, do processo de complexidade do sistema cultural e da sociedade.

Para uma configuração didática deste sistema, podem ser anotados os seguintes momentos, todos eles imprescindíveis ao movimento cultural:

1) Criação, inovação e invenção;

2) Difusão, divulgação e transmissão;

3) Circulação, cooperação, intercâmbios, trocas;

4) Análise, crítica, estudo, investigação, reflexão, pesquisa;

5) Fruição, consumo e públicos;

6) Conservação e preservação;

7) Organização, gestão, legislação e produção da cultura² .

Todos esses momentos do sistema cultural devem ser analisados e articulados em políticas culturais que o compreendam em sua totalidade, desigual e combinada, e formulem propostas para cada um desses momentos e para o conjunto do sistema cultural. As políticas culturais emergem, nesta perspectiva, como conjunto de formulações e práticas que buscam pensar e efetivar o sistema cultural como totalidade integrada.

O sistema cultural não vive sem contradições, as quais derivam de sua inserção no capitalismo, regime dominante na maioria dos países, ou são decorrentes das tensões imanentes do próprio campo da cultura, inclusive as existentes entre os vários momentos do sistema.

Além da diferenciação e especialização das atividades, desenvolvidas através do processo de divisão social do trabalho, será preciso que tais atividades ganhem distinção social. Isto é, sejam reconhecidas pela sociedade. Mais que isso: o reconhecimento social pode se transformar em algo ainda mais substantivo através da confecção de legislações específicas, que regulam a sua prática

2. RUBIM, Antonio Albino Canelas. Marketing cultural. In: RUBIM, Linda (Org.). Organização e produção da cultura. Salvador: EDUFBA, 2005. p. 53-77. e definem exigências para os atores sociais legitimamente encarregados de exercer tais profissões.

Entretanto, a indiferenciação ainda persiste em situações de pouca complexidade do sistema cultural: seja em zonas periféricas da sociedade, seja nas zonas marginais do campo da cultura. Nestes espaços sociais, nem o sistema se 
Organizadores da cultura - Antonio Albino Canelas Rubim e Lindinalva Rubim

desenvolveu plenamente, nem a dinâmica capitalista ainda se instalou de modo profundo. Como se sabe, o capitalismo aprofunda a divisão social do trabalho. Desse modo, nas regiões centrais acontece um adensamento do multifacetado sistema cultural. $\mathrm{O}$ resultado desse processo no presente não poderia deixar de ser um sistema altamente complexo.

\section{OS DIFERENTES PROFISSIONAIS DA CULTURA}

Esse conjunto de atividades e ações, por sua vez, tem exigido da sociedade, através de um longo processo de divisão social do trabalho, a emergência de atores determinados e de profissões especializadas.

Sob este ponto de vista, é possível relacionar as seguintes atividades com os profissionais que foram sendo formatados pela sociedade. A criação cultural está associada aos intelectuais, cientistas, artistas e criadores das manifestações culturais populares. A transmissão, a difusão e a divulgação da cultura constituem o campo, por excelência, dos educadores e professores e, mais recentemente, dos profissionais de comunicação e das mídias. A circulação e o intercâmbio ficam a cargo de mediadores culturais e de outros profissionais competentes na área da cooperação cultural, como diplomatas etc. A preservação e a conservação da cultura - tangível e intangível - requerem arquitetos, restauradores, museólogos, arquivistas, bibliotecários etc. A reflexão e a investigação da cultura são realizadas por críticos culturais, estudiosos e pesquisadores. A organização da cultura solicita formuladores de políticas culturais, gestores, produtores, promotores, programadores e animadores culturais.

A exceção do sistema cultural fica por conta da atividade de consumo, que não demanda uma especialização profissional singular. Ao contrário, a qualidade do sistema pode mesmo ser medida por sua capacidade de ampliar e mesmo universalizar o consumo cultural. Ela supõe que o sistema cultural seja capaz de democratizar a cultura, tornando todos em potenciais e reais consumidores culturais. A complexidade do sistema cultural, neste caso particular, não é medida pela especialização, mas pela capacidade de formação de públicos amplos, que possuam os requisitos e predicados para o consumo e a fruição culturais.

\section{A ORGANIZAÇÃO DA CULTURA}

Diferente do que muitas vezes imagina o senso comum, a cultura é uma atividade, como toda a prática social humana, que requer organização. Uma festa popular como o carnaval, por exemplo, aparentemente lugar, por excelência, do lúdico e da espontaneidade, não pode se realizar sem um imenso esforço de organização. Para a realização de uma festa que mobiliza milhões de pessoas em Salvador, no Rio de Janeiro e em Recife, são necessários imensos recursos financeiros, materiais, técnicos e humanos, além, principalmente, da articulação precisa entre eles. 
3. RUBIM, Antonio Albino Canelas. Espetáculo, política e mídia. In: FRANÇA, Vera et al. (Org.). Livro da XII Compós 2002: estudos de comunicação. Porto Alegre: Sulina 2003. pp. 85-103.

4. ORGANIZAÇÃO DOS ESTADOS IBEROAME RICANOS. Cuadernos Cultura I: conceptos básicos de administración y gestión cultural. Madrid: OEI, 1998. p. 19-20.

5. MADEIRA, Cláudia. Os novos notáveis: os programadores culturais. Oeiras: Celta, 2002.

6. ORGANIZAÇÃO DOS ESTADOS IBEROAMERICANOS. Cuadernos..., cit., p. 19.
O carnaval requer medidas como: organização do cortejo; inscrição das agremiações carnavalescas; ordem e horário das atrações; enfeite e sonorização das ruas e praças; rearranjamento espacial da cidade; bloqueio do trânsito nas vias em que se realiza a festa; policiamento; limpeza; atendimento de saúde; inspeção sanitária; determinação dos serviços de apoio, tais como o comércio de bebidas e de alimentos; construção de arquitetura efêmera para instalar todos estes serviços; ordenação espacial e disposição adequada destes na área da festa; definição da localização dos camarotes; inspeção nos trios elétricos e, enfim, muitas outras tarefas, que não é possível lembrar aqui.

A organização da cultura não é exigida só em manifestações de dimensões espetaculares ou em ações eventuais, mas aparece como obrigatória em atividades permanentes e não tão grandiosas ${ }^{3}$. Um programa televisivo de entrevistas não pode ser efetivado sem que a produção faça todo um trabalho de organização de agenda, considerando as datas significativas, escolha e contato com os convidados, bem como a preparação de roteiros básicos de questões e apresentação dos entrevistados.

Os exemplos do carnaval e do programa de entrevistas na televisão são apenas dois dentre uma infinidade de citações possíveis da necessidade da organização da cultura. Mas tal necessidade não se restringe aos eventos; perpassa todos os processos e, em especial, as estruturas permanentes imprescindíveis ao funcionamento do campo cultural.

A tarefa de organizar a cultura foi realizada historicamente, em outras circunstâncias sociais, por religiosos ou por políticos, pois em grande medida as manifestações da cultura estavam subordinadas a tais esferas. Com a secularização da cultura e sua autonomização enquanto campo social específico, ela passou a solicitar profissionais diferenciados e claramente instalados na sua área.

\section{ORGANIZADORES DA CULTURA: MULTIPLICIDADE DE NOMEAÇÕES}

Uma das características da emergência recente e, em consequência, da ausência de tradição de estudos é a falta de sedimentação, inclusive, na nomeação deste campo. Denominações como: gerentes e administradores culturais predominam nos Estados Unidos e na França; as noções de animadores e promotores culturais possuem uma importante tradição na Espanha; em muitos países da América Latina fala-se em promotores e trabalhadores culturais e, em outros países, podem ser empregados termos como mediadores culturais (França), engenheiros culturais ou científicos culturais ${ }^{4}$. Em Portugal, também se utilizam programadores culturais para dar conta de atividade particular da esfera da organização da cultura ${ }^{5}$. No Brasil, é corrente o uso da noção de produtores culturais. Recentemente, a ideia de gestão cultural ganhou vigência em diversos países, dentre eles os ibero-americanos: "La noción de gestión cultural ingresa al discurso cultural en Iberoamérica con bastante influencia hacia la segunda mitad de la década de los ochenta, tanto en las instituciones gubernamentales como en los grupos culturales comunitarios" ${ }^{\circ}$. 
A pluralidade de denominações não só indica a idade recente das práticas e dos estudos acerca da organização da cultura, como sugere a existência de itinerários e peculiaridades nacionais no que se refere ao desenvolvimento das modalidades organizativas da cultura, com forte incidência sobre a temática da formação de seus profissionais.

\section{SINGULARIDADES BRASILEIRAS}

O caso brasileiro apresenta nesta perspectiva uma notável singularidade, pois a noção predominante tem sido a de produção cultural. Tal dominância pode ser observada na nomeação dos primeiros cursos brasileiros na área de organização da cultura - as graduações em Produção Cultural da Universidade Federal da Bahia e da Universidade Federal Fluminense, surgidas em meados dos anos 1990, e do modo mais usual de (auto)nomeação dos profissionais que trabalham na área. Por exemplo: na dissertação de Maria Helena Melo da Cunha sobre o tema, os profissionais que prestaram depoimentos sobre a construção de seu campo de trabalho na cidade de Belo Horizonte, de preferência, utilizam o termo produtor cultural. Eles só excepcionalmente se reconhecem na expressão gestão cultural, apesar da adesão da autora a este último termo, inclusive no título do trabalho ${ }^{7}$. Entretanto, a opção pela definição de uso mais corrente no Brasil não garante que ela possa ser extensiva ou suficiente a todos os ramos da cultura. Nas artes plásticas ou visuais, as denominações que correspondem às funções de organização são a de curadoria e a de curador. E recentemente têm até mesmo invadido outras manifestações culturais, como a dos organizadores de seminários, cada vez mais chamados de curadores.

A predominância da nomenclatura produção cultural decorre obviamente das peculiaridades históricas, em especial relativas às políticas culturais implantadas no país. Apesar de aquelas de caráter mais sistemático emergirem desde a década de 1930, em especial com a criação do Serviço do Patrimônio Histórico e Artístico Nacional - SPHAN, e de outras instituições culturais no primeiro governo Vargas (1930-1945), o Brasil não desenvolveu, dentre estas políticas, uma preocupação mais abrangente com a formação de pessoal na área de organização da cultura. Não se constituiu no país uma tradição que manifestasse a gestão cultural, enquanto campo específico estimulado pela atuação e pela demanda do Estado nacional.

A ausência de uma política de formação na área da cultura não é uma característica brasileira. A Organização dos Estados Iberoamericanos (OEI), em seu informe sobre cultura e sustentabilidade na Ibero-América, publicado em 2005, afirma: "Sin embargo, parece cierto que una das características del espacio cultural iberoamericano es la indefinición formativo-profesional de los productores, gestores, agentes y mediadores culturales"

Na segunda metade dos anos 1980, o governo José Sarney, de maneira contraditória, ampliou a infraestrutura cultural, inclusive com a criação do Ministério da Cultura em 1985, e, simultaneamente, através da Lei Sarney (1986), deslocou o financiamento da cultura para o mercado, de modo muito
7. CUNHA, Maria Helena Melo da. Gestão cultural: profissão em formação. 2005 (Dissertação de mestrado) - Faculdade de Educação da Universidade Federal de Minas Gerais, Belo Horizonte, 2005.

8. ORGANIZAÇÃO DOS ESTADOS IBEROAMERICANOS. Cultura y sustentabilidad en Iberoamérica. Madrid: OEI/ Fundação Interarts, 2005. p. 10. 
comunicação \& educação • Ano XIV • Número 2 • maio/ago 2009

9. PONTES, Ipojuca. Cultura e modernidade. Brasília: Secretaria de Cultura, 1991.

10. CUNHA, Gestão cultural..., cit.

11. De Gilberto Braga e direção de Dennis Carvalho. Out./2003 a jun./2004.

12. CASTELLO, José. Cultura. In: LAMOUNIER, Bolivar; FIGUEIREDO, Rubens (Orgs.). A era FHC: um balanço. São Paulo: Cultura, 2002. p. 627-656.

13. RUBIM, Linda. Produção cultural. In: RUBIM, Linda (Org.). Organização e produção da cultura. Salvador: EDUFBA, 2005. p. 13-31.

14. RUBIM, Antonio Albino Canelas. Marketing cultural. In: RUBIM, Linda (Org.). Organização e produção da cultura, cit., p. 53-77. particular. Recorrendo a uma política de leis de incentivo, o Estado abriu mão de impostos para que os empresários investissem em cultura. Dessa maneira, o recurso era estatal, mas as decisões passaram a ser do mercado.

As leis de incentivo, em sua modalidade de funcionamento no Brasil, inserem-se na lógica neoliberal, de crítica ao Estado e crença no mercado, que atinge o mundo a partir da virada dos anos 1970/1980, e o Brasil na década de 1990. O governo Fernando Collor (1990-1992) impôs um modelo neoliberal à cultura $^{9}$, com o desmonte das estruturas estatais de cultura, e implantou a Lei Rouanet. De 1992 a 2003, o governo Fernando Henrique Cardoso reformou as leis de incentivo para que tivessem plena vigência em lugar das políticas estatais de cultura, sob a égide do slogan: cultura é um bom negócio.

A lógica de financiamento inscrita nas leis de incentivo possibilitou o surgimento legalizado de intermediários culturais, como seriam chamados na Lei Rouanet. Tais intermediários devem elaborar projetos, captar recursos, administrar eventos etc. Em suma, eles têm de organizar a cultura, em particular, aquela não adstrita ao Estado. Este se encontra quase paralisado, sem recursos próprios para investir e sem políticas culturais, a não ser aquelas relativas à vigência das leis de incentivo.

Não por acaso, Maria Helena Melo da Cunha ${ }^{10}$ constata que a formação do mercado de trabalho de produtores culturais acontece a partir da segunda metade dos anos 1980. Seu reconhecimento social se dá com a telenovela Celebridade $^{11}$, de 2004, veiculada em horário nobre pela Rede Globo. Nela, as duas principais personagens, Maria Clara e Laura, que simbolizam o bem e o mal na narrativa, são produtoras culturais. Profissão recente, pouco conhecida e ainda em processo de constituição, a produção cultural é tornada visível para a grande maioria da população brasileira.

A ausência de tradição na formação de gestores, a submissão da cultura à lógica de mercado e a frágil política cultural do Estado nacional, fortemente intensificadas nos oito anos de Fernando Henrique Cardoso ${ }^{12}$, são, por excelência, o contexto explicativo para a emergência na nomeação de produtores culturais, com as características que eles adquirem no país. Uma discussão cuidadosa sobre a delimitação da figura do produtor cultural pode ser encontrada no trabalho de Linda Rubim ${ }^{13}$. A explicação elaborada elucida igualmente a importância que o marketing cultural obteve no Brasil, comparado com outros países latino-americanos ${ }^{14}$.

\section{A FORMAÇÃO DOS ORGANIZADORES DA CULTURA}

Para além da dificuldade de nomeação, podem ser imaginados três patamares distintos da organização da cultura:

1) a dos formuladores e dirigentes, afeitos ao nível sistemático e macrossocial das políticas culturais;

2) a dos gestores, instalados em instituições ou projetos culturais permanentes, processuais e amplos; e

3) a dos produtores, mais adstritos a projetos de caráter eventual e microssocial. 
Primeira constatação a ser assinalada: a formação em organização da cultura deve compreender, não necessariamente em um único curso ou habilitação, os três patamares aqui registrados, pois é preciso que sejam formados profissionais voltados às políticas, à gestão e à produção cultural, além de outros trabalhadores envolvidos nas diversas dimensões do sistema cultural. A formação para cada um desses patamares, por certo, tem peculiaridades, mas possui muitas características em comum.

De imediato, pode-se constatar o caráter mit [multi-inter-trans] disciplinar deste campo de formação. Além de os conhecimentos acionados serem obrigatoriamente provenientes de uma diversidade de áreas de conhecimento, a própria esfera da cultura e seus estudos já é em si mesma multidisciplinar. A qualificação de organizadores deve trabalhar com um conceito ampliado de cultura, que abarca: ideários, saberes, práticas, comportamentos, valores e uma diversidade de expressões artísticas e científicas. Essa noção ampla de cultura deve ser articulada, de modo íntimo, às mais diferentes áreas de conhecimento: administração, comunicação, economia, educação, história, política, turismo etc. Tais conhecimentos são essenciais para a formação em organização da cultura.

A tentativa de dotar o país de políticas culturais nas quais o Estado tenha papel ativo, como vem acontecendo na gestão Gil/Juca ${ }^{15}$ do Ministério da Cultura*, exige revisar a predominância da produção cultural sobre a gestão e a formulação de políticas culturais, superando a singularidade brasileira, ocasionada pela dominância das leis de incentivo. A formação de formuladores de políticas e gestores culturais passa a ser estruturante para a efetividade das políticas culturais em construção.

Nesta perspectiva, o Brasil poderia seguir o exemplo do Sistema Nacional de Capacitação e Profissionalização de Promotores e Gestores Culturais existente no México, desde 2001, e que até 2005 já tinha possibilitado a graduação de 130 gestores e qualificado mais de 14 mil promotores culturais em outros tipos de $\operatorname{cursos}^{16}$.

A criação de um Sistema Nacional de Formação e Qualificação em Organização da Cultura deveria ser uma das prioridades das políticas culturais brasileiras. A evidente carência nesta área, a ausência de tradição do Estado de investir na capacitação do pessoal da cultura, a reiteração desta demanda nos mais variados encontros de cultura, a exemplo da I Conferência Nacional de Cultura, ocorrida em 2005, além de outros fatores, colocam tal reivindicação como algo essencial para que o país possa dar um salto na implementação qualificada de políticas e no seu desenvolvimento cultural.

Tal sistema deve ser flexível e articular federação, estados, municípios e organizações da sociedade, em especial aquelas dedicadas à formação em cultura, com uma delimitação precisa das responsabilidades que devem caber a cada um destes entes sociais.

Assim, conjugando os esforços do Estado e da sociedade civil, seria possível corrigir as distorções assinaladas da situação brasileira e dotar o país de um programa de formação e qualificação de profissionais em organização da cul-
15. Id. Políticas culturais do Governo Lula/Gil: desafios e enfrentamentos. In: RUBIM, Antonio Albino Canelas; BAYARDO, Rubens (Orgs.) Políticas culturais na lbero-América. Salvador: EDUFBA, 2008. p. 51-74.

* O cantor e compositor Gilberto Gil foi ministro da Cultura do Governo de Luís Inácio Lula da Silva de jan./2003 a jul./2008. O ministério foi assumido por Juca Ferreira, ex-militante estudantil, sociólogo e ambientalista. (N.E.)

16. MAC GREGOR, José Antonio. El papel del coordinador de capacitación cultural dentro del Sistema Nacional de Capacitación y Profesionalización de Promotores y Gestores Culturales de México. In: ORGANIZAÇÃO DOS ESTADOS IBEROAMERICANOS. IV Campus Euroamericano de Cooperación Cultural. Madrid: OEl, 2005. p. 355-359. 
comunicação \& educação • Ano XIV • Número 2 • maio/ago 2009

tura, que, de modo equilibrado, abrangesse os três patamares fundantes desta área: a formulação de políticas, a gestão e a produção cultural.

\section{REFERÊNCIAS BIBLIOGRÁFICAS}

CASTELLO, José. Cultura. In: LAMOUNIER, Bolívar; FIGUEIREDO, Rubens (Orgs.). A era FHC: um balanço. São Paulo: Cultura, 2002.

CUNHA, Maria Helena Melo da. Gestão cultural: profissão em formação. 2005. (Dissertação de mestrado) - Faculdade de Educação da Universidade Federal de Minas Gerais, Belo Horizonte, 2005.

GRAMSCI, Antonio. A formação dos intelectuais. Venda Nova: M. Rodrigues Xavier, 1972.

Literatura e vida nacional. Rio de Janeiro: Civilização Brasileira, 1978.

Os intelectuais e a organização da cultura. Rio de Janeiro: Civilização Brasileira, 1978.

MAC GREGOR, José Antonio. El papel del coordinador de capacitación cultural dentro del Sistema Nacional de Capacitación y Profesionalización de Promotores y Gestores Culturales de México. In: ORGANIZAÇÃO DOS ESTADOS IBEROAMERICANOS. IV Campus Euroamericano de Cooperación Cultural. Madrid: OEI, 2005.

MADEIRA, Cláudia. Os novos notáveis: os programadores culturais. Oeiras: Celta, 2002.

ORGANIZAÇÃO DOS ESTADOS IBEROAMERICANOS. Cuadernos Cultura I. Conceptos básicos de administración y gestión cultural. Madrid: OEI, 1998.

. Cultura y sustentabilidad en Iberoamérica. Madrid: OEI/Fundação Interarts, 2005.

PONTES, Ipojuca. Cultura e modernidade. Brasília: Secretaria de Cultura, 1991.

RUBIM, Antonio Albino Canelas. Espetáculo, política e mídia. In: FRANÇA, Vera et al. (Org.). Livro da XII Compós 2002: estudos de comunicação. Porto Alegre: Sulina, 2003.

. Marketing cultural. In: RUBIM, Linda (Org.). Organização e produção da cultura. Salvador: EDUFBA, 2005.

. Políticas culturais do Governo Lula/Gil: desafios e enfrentamentos. In: RUBIM, Antonio Albino Canelas; BAYARDO, Rubens (Orgs.). Políticas culturais na Ibero-América. Salvador: EDUFBA, 2008.

RUBIM, Linda. Produção cultural. In: RUBIM, Linda (Org.). Organização e produção da cultura. Salvador: EDUFBA, 2005. 